
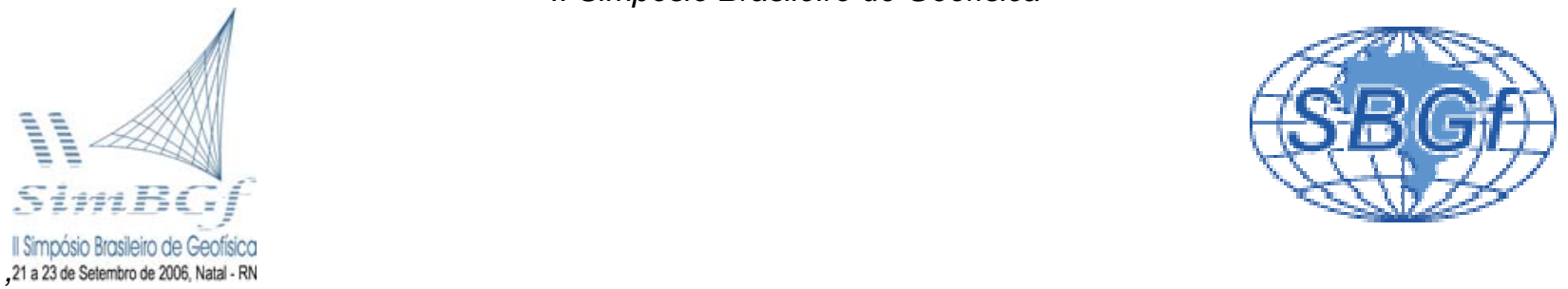

\title{
Inversão por etapas de anomalias magnéticas bidimensionais
}

Soraya Lozada Tuma, Carlos Alberto Mendonça

Instituto de Astronomia, Geofísica e Ciências Atmosféricas, IAG/USP

Copyright 2006, SBGf - Sociedade Brasileira de Geofísica

Este texto foi preparado para a apresentação no II Simpósio de Geofísica da Sociedade Brasileira de Geofísica, Natal, 21-23 de setembro de 2006. Seu conteúdo foi revisado pela Comissão Tecno-científica do II SR-SBGf mas não necessariamente representa a opinião da SBGf ou de seus associados. E proibida a reprodução total ou parcial deste material para propósitos comerciais sem prévia autorização da SBGf.

\section{Abstract}

This work presents a three step magnetic inversion procedure in which invariant quantities related to source parameters are sequentially inverted to provide i) crosssection of two-dimensional sources; ii) intensity of source magnetization, and iii) inclination of source magnetization. The first inverted quantity (called geometrical function) is obtained by rationing the gradient intensity of the total field anomaly by the anomalous field intensity. For homogenous sources, geometrical function depends only on source geometry, thus allowing shape reconstruction by using arbitrary values for source magnetization. Source shape is then fixed and magnetization intensity is estimated by fitting the gradient intensity of the total field anomaly. In its last step, source shape and magnetization intensity are fixed and magnetization inclination is determined by fitting the magnetic anomaly. The proposed technique is tested with a numerical experiment, and implications further discussed.

\section{Introdução}

A idéia básica na interpretação quantitativa de dados de campo potencial (gravimetria e magnetometria) envolve a representação das fontes do substrato por um modelo geométrico, com propriedades físicas definidas (ou a serem obtidas), cuja anomalia teórica seja compatível com a anomalia medida em campo. A anomalia correspondente ao modelo deve ajustar a anomalia medida ou, pelo menos, representar algumas de suas feições mais expressivas. Além de propiciar o ajuste aos dados, o modelo geofísico deve ser fisicamente plausível e incorporar informações geológicas disponíveis ao intérprete.

Via de regra, a interpretação de anomalias magnéticas assume que a direção de magnetização seja conhecida. Esta premissa pode ser bastante restritiva quando se consideram fontes profundas não amostradas ou com forte remanescência.

O presente estudo explora a noção de invariância seletiva de campos magnéticos em relação a parâmetros da fonte para delinear um procedimento de inversão por etapas no qual se determina, em sequência, a geometria da fonte e sua magnetização. Seu ponto de partida é a definição de uma função invariante com o vetor de magnetização, mas dependente da geometria da fonte. Esta função, denominada G (por depender apenas da geometria) é definida pela razão

$$
\mathrm{G}=\frac{\mathrm{A}}{\mathrm{T}}
$$

sendo A, a amplitude do sinal analítico (Nabighian, 1972) e T, a intensidade do campo magnético anômalo (Stavrev \& Gerovska, 2000). Para fontes 2-D, estas grandezas independem da direção da magnetização, permitindo a determinação de parâmetros relacionados à geometria das fontes, mesmo quando se desconhece o valor desta propriedade física.

Para calcular G, é necessário calcular inicialmente as grandezas A e $\mathrm{T}$. O cálculo de A requer as primeiras derivadas x e $\mathrm{z}$ da anomalia. $\mathrm{O}$ cálculo de $\mathrm{T}$ requer as componentes x e z do campo vetorial anômalo.

\section{Formulação do problema inverso}

Nesta formulação, vamos representar a fonte magnética por um conjunto $\mathrm{n}_{\mathrm{p}}$ de prismas 2-D justapostos, homogêneos. Os parâmetros desconhecidos são: bordas esquerda, $x_{i}$, e direita, $x_{f}$, do conjunto de prismas ao longo do eixo $x$, os topos, $t_{i}$, e as bases, $b_{i}, i=1,2, \ldots, n_{p}$ de cada prisma, a inclinação da magnetização, $\tau$ e a intensidade da magnetização, M. Estes parâmetros são arranjados em um vetor transposto $\mathrm{p}^{\mathrm{T}}=\left[\mathrm{x}_{\mathrm{i}}, \mathrm{x}_{\mathrm{f}}, \mathrm{t}_{1}, \mathrm{t}_{2}, \ldots, \mathrm{t}_{\mathrm{np}}, \mathrm{b}_{1}, \mathrm{~b}_{2}, \ldots, \mathrm{b}_{\mathrm{np}}, \tau, \mathrm{M}\right]$ de dimensão $\mathrm{n}=2 \mathrm{n}_{\mathrm{p}}+4$, sendo $\mathrm{p}_{\mathrm{j}} \mathrm{o}$ j-ésimo parâmetro desconhecido do conjunto de prismas. Aos dois últimos elementos desse vetor, que definem a inclinação e a intensidade de magnetização da fonte, são atribuídos valores arbitrários.

\section{Etapa 1 - Geometria das fontes}

Para determinar a geometria das fontes utilizamos o algoritmo CRS - Controlled Random Search (Price,1977) para estimar os primeiros $2 n_{p}+2$ parâmetros de $\mathbf{p}$. O CRS utiliza como entrada $N$ soluções iniciais que foram geradas perturbando uma solução de partida, $\mathbf{p}_{\mathbf{o}}$, que incorpora vínculos de profundidade para o topo e a base. $\mathrm{O}$ funcional $\mathrm{Q}(\mathbf{p})$ minimizado é composto por três parcelas: 
$\mathrm{Q}(\mathbf{p})=\frac{1}{\mathrm{~V}_{\mathrm{r}}} \sum_{1}^{\mathrm{n}_{\mathrm{d}}}\left(\mathrm{G}_{\mathrm{i}}^{0}-\mathrm{G}(\mathrm{p})_{\mathrm{i}}\right)^{2}+\frac{\mu_{\mathrm{r}}}{\mathrm{P}_{\mathrm{r}}} \sum_{\mathrm{j}=\mathrm{J}_{\mathrm{i}}}^{\mathrm{J}_{\mathrm{f}}}\left(\mathrm{p}_{\mathrm{j}+1}-\mathrm{P}_{\mathrm{j}}\right)^{2}+\frac{\mu_{\mathrm{a}}}{\mathrm{P}_{\mathrm{r}}} \sum_{\mathrm{j}=1}^{\mathrm{n}_{\mathrm{W}}}\left(\mathrm{p}_{\mathrm{k}}-v_{\mathrm{k}}\right)_{\mathrm{k} \equiv \mathrm{k}(\mathrm{j})}^{2}$

A primeira parcela propicia o ajuste da função geométrica para $\mathrm{n}_{\mathrm{d}}$ dados; a segunda impõe o vínculo de proximidade relativa para os parâmetros que definem a base do modelo, parâmetros de índice $\mathrm{J}_{\mathrm{i}}=\mathrm{n}_{\mathrm{p}} / 2+1$ a $\mathrm{J}_{\mathrm{f}}=2\left(\mathrm{n}_{\mathrm{p}}+1\right)$. Na formulação adotada, o vínculo de proximidade relativa não foi aplicado na definição da topografia do topo. Finalmente, a terceira parcela impõe vínculos de proximidade absoluta para $\mathrm{n}_{\mathrm{w}}$ pontos com profundidades $v_{\mathrm{k}(1)}, v_{\mathrm{k}(2)}, \cdots, v_{\mathrm{k}\left(\mathrm{n}_{\mathrm{w}}\right)}$ conhecidas por afloramento, sondagem ou outras investigações geofísicas. Os elementos $\mu_{\mathrm{r}}$ e $\mu_{\mathrm{a}}$ são multiplicadores de Lagrange (números reais positivos). Os parâmetros $\mathrm{V}_{\mathrm{r}} \mathrm{e}$ $\mathrm{P}_{\mathrm{r}}$ normalizam as parcelas de $\mathrm{Q}(\mathbf{p})$. O parâmetro $\mathrm{P}_{\mathrm{r}}$, adicionalmente, representa uma profundidade de referência para o parâmetro da base.

\section{Etapa 2- Intensidade de magnetização}

Nesta etapa, a inversão fixa a geometria obtida na etapa anterior e determina a intensidade de magnetização a partir do ajuste da amplitude do sinal analítico A . Fixando a geometria das fontes, calculam-se os valores teóricos da amplitude do sinal analítico para uma magnetização de $1 \mathrm{~A} / \mathrm{m}$. No caso de magnetização uniforme, os valores teóricos e medidos diferem apenas por um fator constante, que define a intensidade de magnetização $\bar{M}$.

\section{Etapa 3 - Inclinação da magnetização}

Nesta etapa, os resultados anteriormente obtidos (geometria da fonte e a intensidade de magnetização) são fixados e usados para determinar a inclinação da magnetização a partir do ajuste da anomalia magnética. Configura-se, assim, um problema inverso no qual $n_{d}$ valores da anomalia magnética são ajustados a partir da variação de um parâmetro apenas: a inclinação da magnetização. Para tanto, define-se um funcional $\mathrm{R}(\tau)$, dependente apenas da inclinação de magnetização, $\tau$, tal que:

$\mathrm{R}(\tau)=\sum_{\mathrm{i}=1}^{\mathrm{n}_{\mathrm{d}}}\left(\mathrm{T}_{\mathrm{i}}^{\mathrm{o}}-\mathrm{T}_{\mathrm{i}}^{\mathrm{c}}\left(\tau_{\mathrm{k}}\right)\right)^{2}$

sendo $\mathrm{T}_{\mathrm{i}}{ }^{0}$ o valor da anomalia magnética na i-ésima posição, $\quad \mathrm{T}_{\mathrm{i}}^{\mathrm{c}}\left(\tau_{\mathrm{k}}\right) \equiv \mathrm{T}_{\mathrm{i}}{ }^{\mathrm{c}}\left(\overline{\mathbf{s}}, \overline{\mathrm{M}}, \tau_{\mathrm{k}}\right) \quad$ o valor calculado na mesma posição assumindo o modelo definido pela solução $\overline{\mathbf{s}}$ (parâmetros da geometria estimados na etapa
1) com intensidade de magnetização $\bar{M}$ (etapa 2) e inclinação de magnetização $\tau_{\mathbf{k}}$.

\section{Experimento numérico}

A inversão por etapas é utilizada neste experimento para recuperar o relevo do topo de uma fonte magnética com espessura infinita. O modelo verdadeiro é formado por dez prismas justapostos, com magnetização de 700 $\mathrm{mA} / \mathrm{m}$, na direção de $-70^{\circ}$. As Figuras 1 e 2 mostram passos intermediários do procedimento de inversão por etapas e a Figura 3 mostra os resultados do teste.

A Figura 1 mostra a estimativa da intensidade da magnetização, sendo que a mediana das estimativas $(686 \mathrm{~mA} / \mathrm{m})$ é próxima ao valor verdadeiro $(700 \mathrm{~mA} / \mathrm{m})$. Como a fonte é homogênea, as estimativas da intensidade da magnetização determinam um patamar cuja mediana ajusta satisfatoriamente a amplitude do sinal analítico (Figura 1c).

A Figura 2 ilustra a determinação da inclinação da magnetização usando o método de varredura. Observase que a inclinação da magnetização verdadeira coincide com o valor estimado de $-70^{\circ}$ (Figura 2a). A inclinação estimada ajusta a anomalia magnética.

A Figura 3 apresenta o modelo invertido e as barras de erro associadas a cada parâmetro. Observa-se que o modelo estimado coincide com o modelo verdadeiro. Como não foi utilizada informação a priori, o resultado do teste indica que a função geométrica tem informação suficiente para recuperar o relevo do topo (no caso, representado por uma forma em degrau) e posicionar as bordas. A faixa estreita para as barras de erro mostra que a solução obtida é estável.

\section{Conclusões}

A inversão por etapas permitiu obter uma solução para o problema inverso em magnetometria sem exigir informação a priori a respeito magnetização da fonte. Isto ilustra sua potencialidade na inversão de anomalias causadas por fontes com magnetização desconhecida e com forte remanescência. A geometria da fonte, no caso definida por uma interface apenas, também foi satisfatoriamente delineada.

\section{Agradecimentos}

Ao CNPQ/PEC-PG. Ao Instituto de Astronomia, Geofísica e Ciências Atmosféricas, IAG/USP. À Embaixada do Brasil, ao Instituto Brasileiro Equatoriano de Cultura, IBEC, Quito-Equador. 

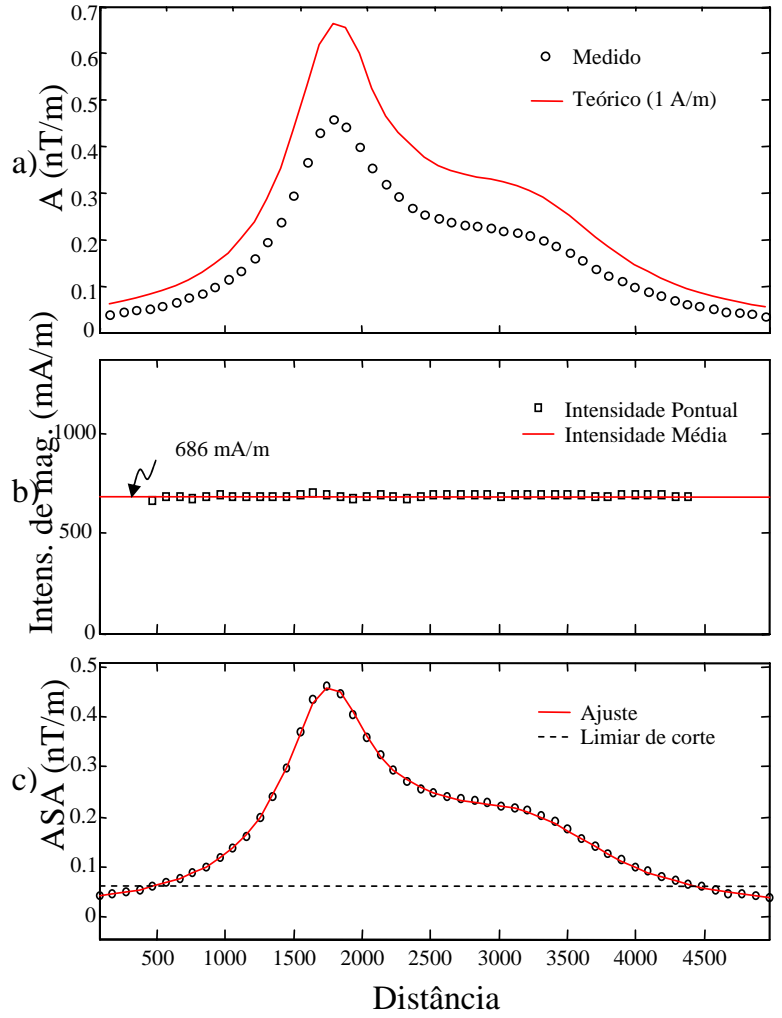

Figura 1 -llustração do procedimento de inversão por etapas: Etapa 2 da inversão por etapas: a) Valores de $A$ medidos (círculos) e teóricos assumindo modelo prismático obtido na Etapa 1, com magnetização de $1 \mathrm{~A} / \mathrm{m}$. b) intensidade de magnetização obtida pela razão dos dois campos na figura superior. c) Ajuste aos dados de $A$ utilizando intensidade de magnetização de 686 $\mathrm{mA} / \mathrm{m}$. Pontos menores que o valor de corte (tracejado azul) não foram considerados no cálculo da intensidade média.
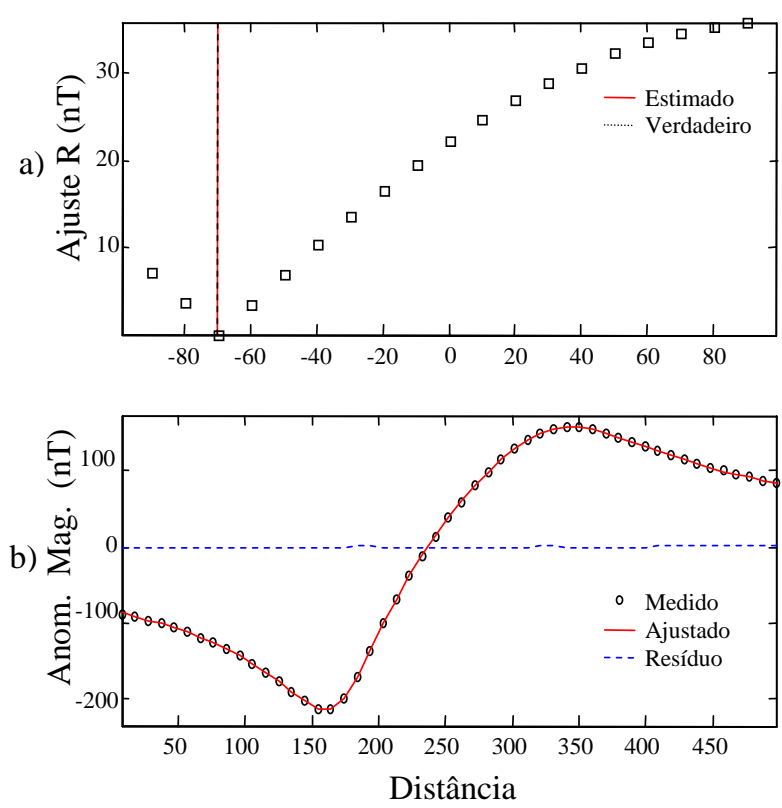

Figura 2 - llustração do procedimento de inversão por etapas. Etapa 3: determinação da inclinação da magnetização por busca direta (algoritmo de varredura): a) busca preliminar usando passo de $1^{\circ}$. b) anomalia magnética calculada usando inclinação de $-70^{\circ}$ (ponto mínimo da curva de varredura).

\section{Referências}

Nabighian, M. N., 1972, The analytic signal of twodimensional magnetic bodies with polygonal cross section: Its properties and use for automated anomaly interpretation: Geophysics, 37, 507-517.

Price, W. L., 1977, A controlled random search procedure for global optimization: The Computer Journal,20, 357370.

Stavrev, P. \& Gerovska, D., 2000, Magnetic field transforms with low sensitivity to the direction of source magnetization and high centricity: Geophysical Prospecting, 48, $317-340$. 
a)

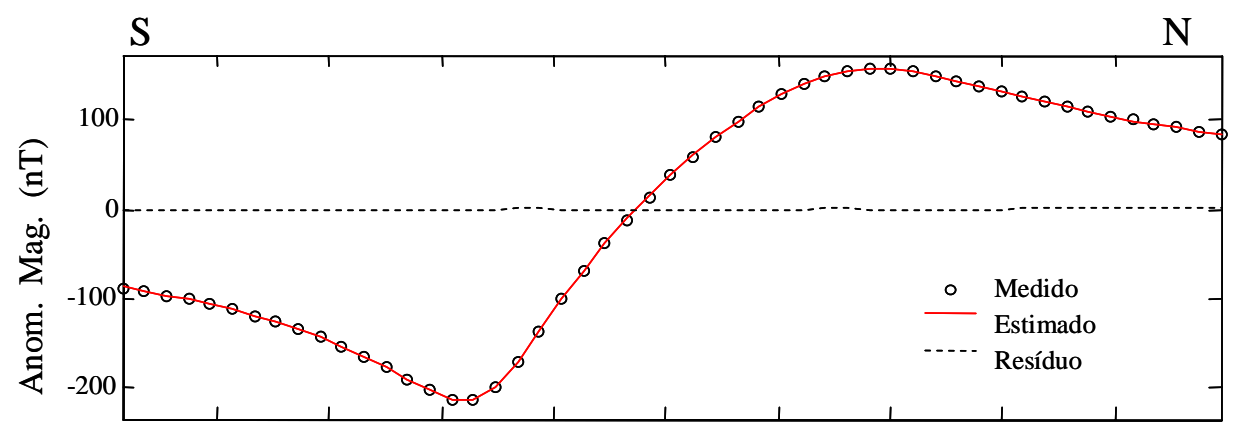

b)
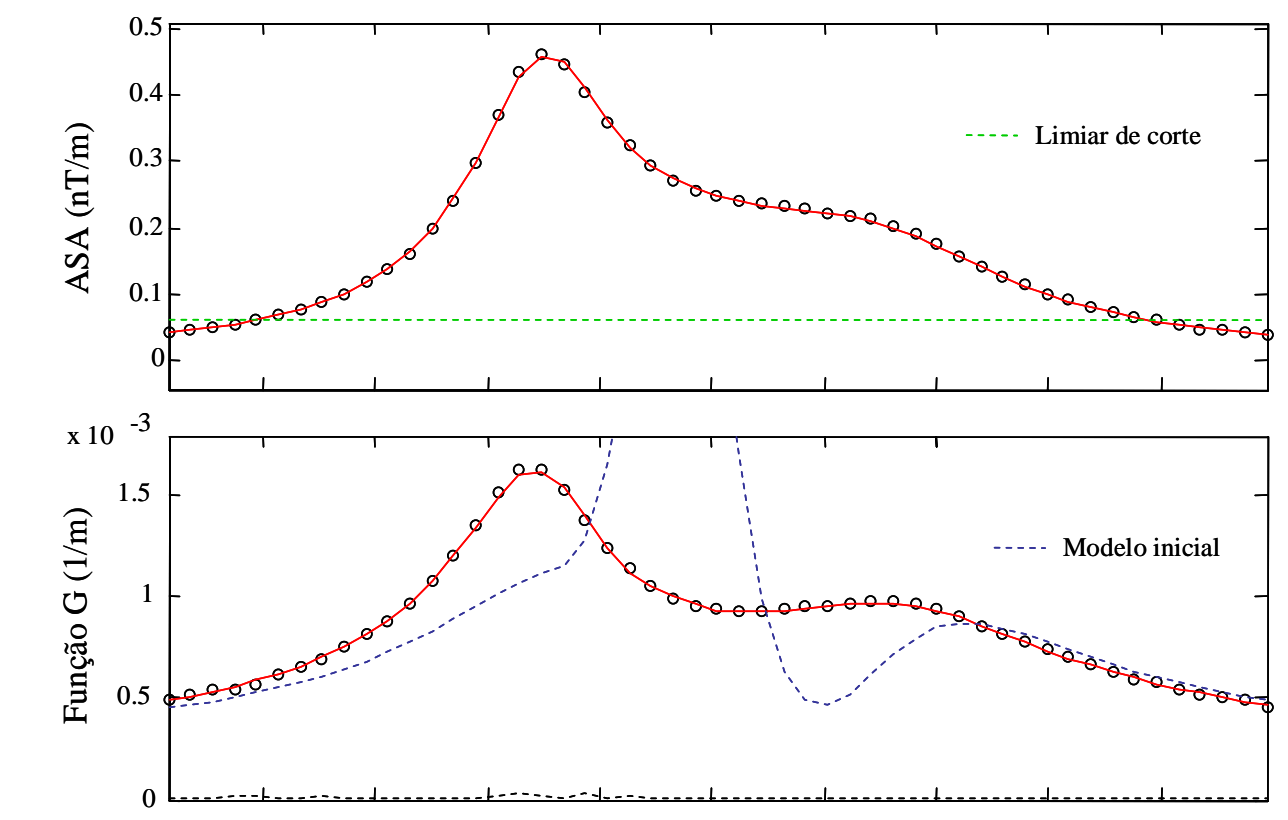

c)

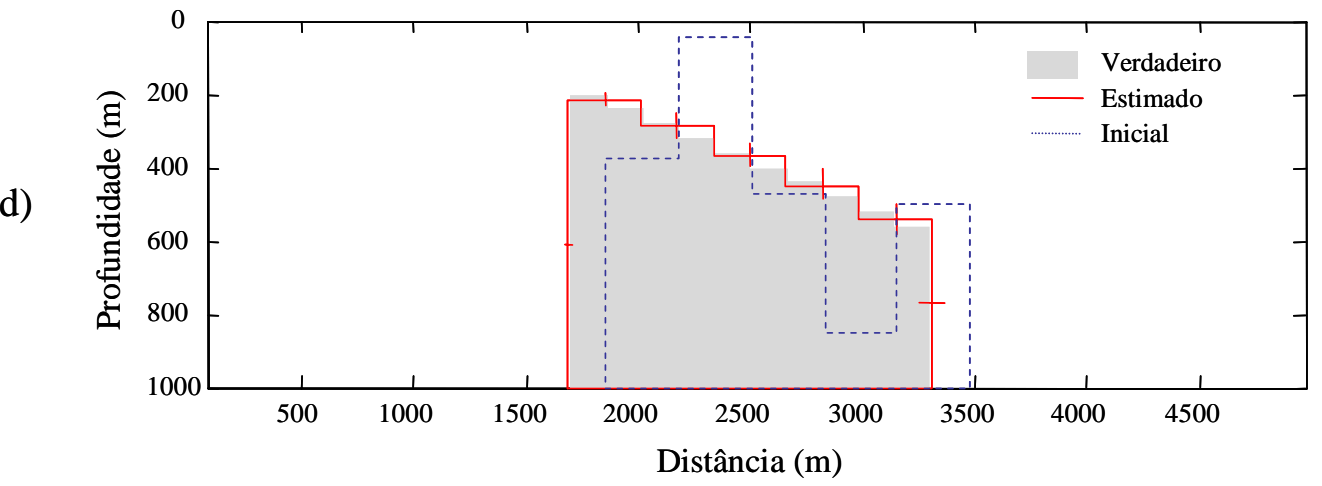

Figura 3 - Mapeamento do topo de fontes magnéticas com espessura infinita (topo com mergulho norte); a) anomalia magnética sintética (círculo) e ajustada (vermelho); b) ASA sintética (círculo) e ajustada (vermelho); c) função geométrica sintética (círculo) e ajustada (vermelho). A e G sintéticas foram obtidas por processamento da anomalia magnética no nível de $100 \mathrm{~m}$. A anomalia contem $0.2 \%$ de ruído aleatório. Parâmetros de inversão: modelo com $\mathrm{n}_{\mathrm{p}}=5, \mu_{\mathrm{a}}=0 ; \mu_{\mathrm{r}}=0, \mathrm{~N}=60$ e $\mathrm{v}_{\mathrm{r}}=0,23$. Parâmetros estimados: intensidade de magnetização de $686 \mathrm{~mA} / \mathrm{m}$, inclinação da magnetização de $70^{\circ}$. Desvio padrão da intensidade, +-7,4 mA/m. Modelo verdadeiro composto por 10 prismas; magnetização com intensidade de $700 \mathrm{~mA} / \mathrm{m}$ e inclinação de $-70^{\circ}$. Campo geomagnético com inclinação de $-18^{\circ}$ e declinação nula. 